\title{
Optimiser la combustion : les nouvelles méthodes optiques pour étudier l'aérodynamique interne des moteurs
}

\author{
La vélocimétrie par image de particules (ou PIV), mise en œuvre dans un moteur expérimental doté d'accès \\ optiques, se révèle d'une utilité cruciale dans l'étude des écoulements internes turbulent dans les cylindres. \\ Des outils récents tels que FTLE ou POD invariante en phase permettent de mieux connaître les écoulements dits \\ «de rouleau» et surtout d'appréhender les fluctuations cycle à cycle associées. Appliquées aux mesures \\ expérimentales aussi bien qu'aux prévisions numériques, ces méthodes sont en passe de s'imposer comme des \\ références pour l'industrie.
}

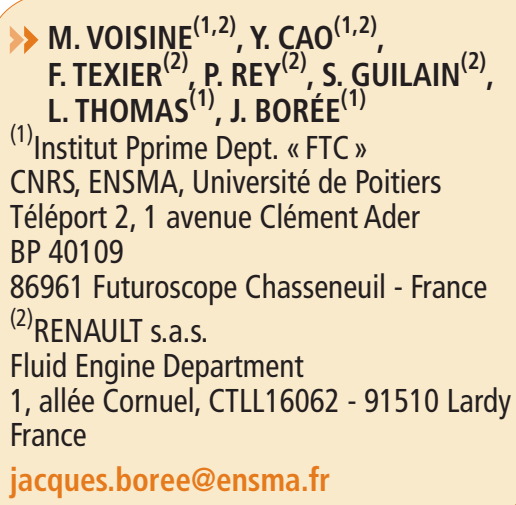

\section{Introduction}

La connaissance et la maîtrise des écoulements dans les cylindres fait partie des enjeux actuels du secteur automobile car ils exercent un rôle majeur sur la préparation du mélange air/carburant et sur le déroulement de la combustion (Lumley, 1999). L'évolution des chambres de combustion des moteurs, sous la forte contrainte de normes de pollution de plus en plus sévères, requiert un contrôle précis de la préparation et de la dynamique du mélange avant la phase de combustion. Il est alors extrêmement important de pouvoir comprendre et maîtriser les écoulements internes, leur génération pendant l'admission et leur évolution pendant la compression. Les questions fondamentales concernent le comportement dynamique et la stabilité de ces écoulements, l'évolution de la turbulence soumise à ces mouvements et bien sûr l'évolution du champ de mé- lange. La demande des constructeurs est fortement liée à l'émergence de technologies nouvelles. Les nouvelles stratégies exigent souvent la maîtrise de la composition du mélange dans le cylindre ou autour de la bougie à l'instant d'allumage tout en accordant une grande importance à la stabilité cyclique.

Bien que l'importance des écoulements internes au moteur ait été reconnue très tôt, il a fallu attendre le développement des techniques de diagnostics optiques et en particulier de l'anémométrie laserDoppler dans les années 70 pour obtenir des mesures détaillées dans les chambres de combustion. En général, ces mesures ponctuelles permettent d'obtenir une ou deux composantes de la vitesse en quelques points du cylindre seulement. Il est donc très difficile de comprendre la structure de l'écoulement et son évolution. La vélocimétrie par image de particules (PIV), développée au début des années 90 , a connu un essor considérable. Les mesures par PIV en moteur transparent entraîné sont maintenant relativement courantes et bien adaptées à la résolution du champ instantané. Chez les constructeurs, la PIV est ainsi en passe de devenir un outil de dimensionnement efficace et permet de renforcer l'interaction entre mesures et modèles de prévision numérique.

Le but de la présente étude est d'utiliser à la fois la PIV et la PIV rapide dans un moteur de recherche disposant de larges accès optiques de façon à étudier la structure spatiale d'un écoulement de rou- leau, mouvement de rotation à grande échelle dans le cylindre généré pendant la phase d'admission, et son évolution temporelle lors de phases critiques du cycle. Son axe de rotation principal est perpendiculaire à l'axe du cylindre. L'intérêt est ici de stocker l'énergie cinétique des jets de soupapes dans une structure à grande échelle moins dissipative. L'instabilité de cette structure en fin de compression se traduit par une « rupture » du rouleau et par un transfert vers une turbulence à fine échelle importante pour la phase de combustion ultérieure.

\section{Le banc moteur et les méthodes de mesures}

Les expériences sont menées au centre de Lardy de Renault SA dans un moteur monocylindre 4 soupapes entraîné par une génératrice électrique. La culasse en toit a un angle de $28^{\circ}$, la course est de $73,1 \mathrm{~mm}$ pour un alésage de $78 \mathrm{~mm}$. La pression maximale au point mort haut (fin de la compression) est de 18 bars. Le cylindre est transparent pour permettre la mesure par méthode optique. Le piston est plat, transparent et muni d'une rehausse. En effet, il est possible de visualiser l'intérieur du cylindre ou de faire passer une nappe laser à travers le piston via un miroir de renvoi situé sous le piston. Maîtrise de l'étanchéité et réduction des volumes morts sont des points clés lors de la conception de tels systèmes. Un effort particulier a été consacré à la réalisation d'un hublot sur $\gg$ 
un côté de la chambre en toit, permettant de visualiser l'écoulement jusqu'à la fin de la compression. Un régime de rotation de $1200 \mathrm{tr} / \mathrm{min}$ et une pression à l'admission proche de la pression atmosphérique ont été sélectionnés pour ces essais. Un système de codeur angulaire permet de connaître les degrés vilebrequin (DV) et de piloter la mesure.

La mesure par PIV revient à mesurer le déplacement de fines particules ensemençant l'écoulement entre deux tirs laser séparés d'un intervalle de temps choisi avec soin. Deux composantes de la vitesse dans le plan de mesure sont obtenues ici. Nous avons vérifié que les particules solides d'ensemencement suivent bien toutes les échelles de temps de l'écoulement (Cosadia et al., 2006). Un système «Dantec » et un laser à deux cavités Nd:Yag (100 mJ/pulse) sont utilisés pour obtenir des statistiques dites « de phase ", calculées pour un angle vilebrequin choisi. Les images sont enregistrées par une caméra CCD «Flowsense» de $1600 \times 1200$ pixels $^{2}$ et une taille de $0,073 \mathrm{~mm} /$ pixel dans le plan objet pour la plupart des situations. Le détail des paramètres du traitement PIV est donné dans (Voisine et al., 2011). Notez que l'erreur absolue estimée sur la mesure du déplacement est classiquement de 0.1 pixel. Avec un laser $\mathrm{Nd}$ :Yag tirant à $10 \mathrm{~Hz}$ et un régime moteur de $1200 \mathrm{tr} /$ min, il est facile de vérifier qu'une mesure par cycle est obtenue pour un DV choisi. 500 cycles consécutifs sont mesurés pour le calcul des moyennes, des variances et des corrélations entre composantes fluctuantes de vitesse (constituants du tenseur de Reynolds).

Un système « LaVision » associé à un laser Litron LDY303, délivrant deux pulses consécutifs (séparés ici de $30 \mu$ s) d'une puissance de $10 \mathrm{~mJ}$ à une fréquence de $1,8 \mathrm{kHz}$, est utilisé pour les mesures PIV rapides.

Les images sont enregistrées par une caméra CMOS HSS6 de $1024 \times 1024$ pixels ${ }^{2}$.

Avec un double tir laser tous les 4 DV et une résolution spatiale de l'ordre de $2 \mathrm{~mm}$, il serait illusoire de prétendre résoudre toutes les échelles temporelles et spatiales de cet écoulement turbulent. Toutefois, il est possible de suivre les mouvements à grande échelle dans le cylindre dans une portion du cycle. Nous l'avons fait dans le plan de symétrie en fin d'admission et dans la chambre en toit en fin de compression. Soulignons qu'une difficulté importante dans la réussite de telles mesures par PIV rapide est la maîtrise de l'ensemencement dans un volume variant fortement au long du cycle. Développements et mises au point sont donc très longs avant de parvenir à une mesure fiable.

\section{Structuration de l'écoulement pendant l'admission et le début de compression}

Pour comprendre la chaîne d'événements conduisant à la structuration du rouleau, nous avons d'abord associé des mesures PIV en moyenne de phase dans un ensemble de plans de mesure. La structure des conduits d'admission fait que la combinaison des jets de soupapes longe la paroi opposée du cylindre. Le flux de quantité de mouvement résultant impacte alors le piston, longe sa surface et remonte le long des parois du cylindre. Une fois les soupapes d'admission fermées, la structure moyenne de l'écoulement est donc complexe et fortement tridimensionnelle, comme le montre la figure 1 obtenue au milieu de la phase de compression. L'intérêt de la PIV est toutefois de fournir des familles de champs spatiaux instantanés. Les statistiques de quantités intégrales (circulations, flux...) ont donc été calculées (Voisine et al., 2011) et prouvent une forte variation cycle à cycle de structure et d'intensité de l'écoulement au point mort bas, corrélée au développement des jets de soupapes. Une fois les soupapes fermées, il est alors évident que c'est le développement de la structure à grande échelle dans le cycle considéré qui est important pour les performances du moteur. L'utilisation de la PIV rapide est alors très importante.

\section{Variations cycliques pendant la phase de compression}

Deux séquences particulières de la « vie » du rouleau ont ainsi été considérées. La position et la propagation du front du rouleau, longeant la paroi du cylindre en début de phase de compression

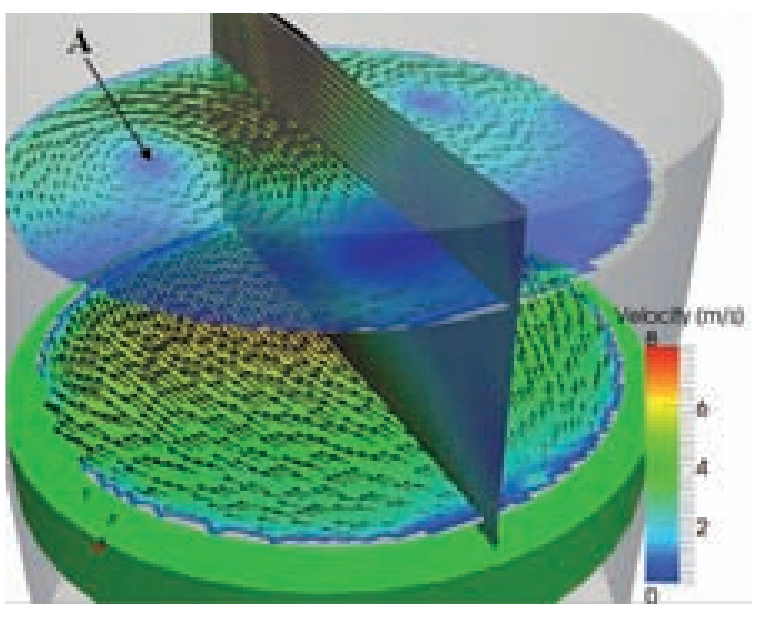

Figure 1. Structure tridimensionnelle de l'écoulement moyen au milieu de la phase de compression (270 DV).

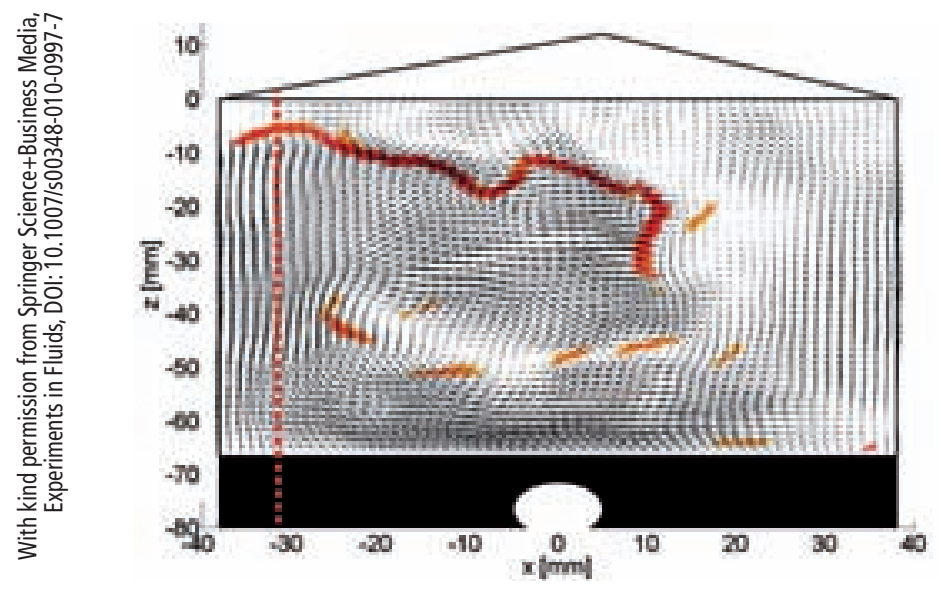

Figure 2. Champ instantané et crête des FTLE à 220 DV. Ce front permet d'extraire la propagation du front du rouleau des données PIV rapide. 
(figure 2), a d'abord été étudiée. D’un point de vue topologique, ce front correspond à la branche instable d'un point selle si l'on se place dans un référentiel en mouvement avec l'écoulement. II a donc été détecté en calculant les champs d'exposants de Lyapounov à temps fini (FTLE - Haller, 2001) à partir des données de PIV rapide - voir les détails de mise en œuvre dans (Voisine et al., 2011).

Nous avons ainsi mis en évidence une très forte variation cyclique de la position du front du jet (de l'ordre de $10 \%$ de la course du piston), fortement corrélée aux variations des propriétés dynamiques du rouleau. À notre connaissance, ces techniques d'analyse récentes sont appliquées ici pour la première fois en moteur. Elles sont intéressantes pour explorer la cohérence spatiale et temporelle des écoulements internes et leur variation cycle à cycle.

Dans le même esprit, la phase cruciale de rupture du rouleau en fin de compression a été caractérisée en utilisant des données de PIV rapide résolues tous les 4 DV de 270 DV à 360 DV (le point mort haut). Une analyse classique de moyenne de phase montre que l'énergie cinétique moyenne du rouleau décline en un temps de retournement après $300 \mathrm{DV}$, ce qui est classiquement interprété comme la phase de rupture. Toutefois, I'augmentation de l'énergie cinétique turbulente alors mesurée mélange en fait deux aspects physiques très différents: d'une part un transfert vers une turbulence à fine échelle ayant une influence sur la phase de combustion ultérieure dans le cycle considéré ; d'autre part une variation d'un cycle à l'autre de la structure de l'écoulement. Une extension de la décomposition orthogonale propre (Fogleman et al., 2004) (POD pour les anglophones, similaire à I'analyse en composante principale bien connue en statistique) a été appliquée aux données PIV rapide car elle permet d'extraire de façon optimale la cohérence spatiale et temporelle de l'écoulement mesuré. Les cycles mesurés par PIV rapide (161 dans le cas présent) peuvent alors être classés suivant leur structure au point mort haut, ce qui permet le calcul et l'analyse physique de statistiques conditionnelles pour l'évolution de différentes familles de cycles au cours de la compression. Le détail des résultats n'est pas donné ici mais retenons qu'au voisinage de la fin de compression dans cette chambre modèle, les fluctuations de structure d'un cycle à l'autre sont responsables d'un tiers de l'énergie cinétique fluctuante détectée en moyenne de phase.

\section{Conclusion}

Pour ces écoulements complexes au cœur des préoccupations sociétales, le premier défi est d'obtenir des bases de données expérimentales et numériques fiables, autorisant l'analyse. À ce titre, les enjeux associés à la maitrise des fluctuations cycle-à-cycle sont des moteurs puissants pour le développement de métrologies PIV rapides ou pour la mise au point de modélisations hybrides ou aux grandes échelles de ces écoulements (Vermorel et al., 2009). L'analyse des énormes quantités de données associées est également un défi en soi pour la communauté scientifique et technique. Nous avons donc insisté dans ce travail sur I'application d'outils récents (FTLE, POD invariante en phase) qui permettent d'identifier, de façon quantitative et objective, les structures d'écoulements responsables des variations cycliques. Le développement de ces méthodes appliquées tant aux données expérimentales qu'aux données numériques sera in fine utile d'un point de vue technique pour quantifier objectivement la robustesse d'un concept moteur.

\section{Références \\ Cosadia, I., J. Borée, G. Charnay and P. Dumont (2006). «Cyclic variations of the swirling flow in a Diesel trans- parent engine. » Exp. in Fluids 41:115-134. \\ Fogleman, M., D. Rempfer, J.L. Lumley and D. Haworth (2004). «Analysis of tumble breakdown in IC engine flows using the POD.» Journal of Turbulence 5: 023(http://jot.iop.org/). \\ Haller, G. (2001). « Distinguished material surfaces and coherent structures in three dimensional fluid flows. » Physica D 149: 248-277.}

Lumley, J.L. (1999). Engines. An introduction, Cambridge University Press.

Vermorel, 0., S. Richard, O. Colin, C. Angelberger, A. Benkenida and D. Veynante (2009). «Towards the understanding of cyclic variability in a spark ignited engine using multi-cycle LES. » Combust. Flame 156: 1525-1541.

Voisine, M., L. Thomas, J. Borée and P. Rey (2011). "Spatio-temporal structure and cycle to cycle variations of an in-cylinder tumbling flow. » Exp. in Fluids, DOI 10.1007/s00348-010-0997-7.

\section{PV Direct Nouvelle revue internationale open access}

Destinée aux acteurs (chercheurs, ingénieurs, etc.) dans le domaine photovoltaique, PV direct publie des articles originaux dans les différents secteurs de la conversion de l'énergie solaire: modules, composants, systèmes...

Composée d'un comité éditorial international, sa formule "open access" lui offre de nombreux avantages : grande visibilité, rapidité de publication, figures couleurs...

Pour plus d'information sur cette revue et envoyer vos articles, visitez le site internet 